\title{
Determinism vs freedom: some ethics-social implications
}

\section{[Determinismo vs libertad: algunas implicaciones etico-sociales]}

\author{
Marian Ambrozy - Roman Kralik - Jose Garcia Martin
}

\section{DOI: $10.18355 /$ XL.2017.10.04.05}

\begin{abstract}
Resumen
El artículo es un intento de considerar brevemente el modo de comprender el propósito de la libertad en términos de desarrollo del status en la física de los objetos observados. En primer lugar, trata de definir conceptos tales como "determinismo", "indeterminismo", "casualidad" y "predictibilidad". Luego trata de caracterizar el tema de la predictibilidad al nivel del caos determinístico. Más tarde, el mismo tema se aplica en el campo del microcosmos, esto es, en la teoría cuántica. Todo lo cual trata de responder a nuestra cuestión en conformidad con la ambivalencia de los resultados de la investigación. A su vez, se trata de dilucidar algunas consecuencias con relación a la ética, las creencias religiosas y la libertad. Finalmente, se hace una revisión crítica del problema sobre fe y razón.
\end{abstract}

Palabras clave: ética, religión, libertad, fe, razón

Introduction

El caos es un tema ampliamente debatido hoy en día. Anteriormente, el caos estaba asociado principalmente a valoraciones negativas. Tenía su lugar importante en la mitología. Sin embargo, en otras esferas, tales como las ciencias naturales y sociales, se percibía negativamente. En las ciencias formales el término caos casi equivalía a pseudociencia. Naturalmente, estos diferentes planteamientos exigen cierta uniformidad terminológica en el campo científico: "Specialists include continuous defining and consensus based determining of unified terminology" (Vintar, Simeunoviæ, 2017: 43-58). Tampoco la teología, la ciencia de lo sobrenatural, percibía el caos desde una perspectiva positiva. La teología supone que el desorden y el caos están asociados con el mal y con su personificación, Satanás.

A nivel macroscópico, existe el llamado caos determinista. El movimiento caótico puede ser libremente caracterizado de la siguiente manera: "Deterministic chaotic models have been largely studied in the past few decades, both on the numerical and theoretical sides". (Gissinger, 2012: 137).

El caos determinista como tal surge en dos tipos de sistemas. Por un lado, hallamos los sistemas no disipativos, conservativos. Estos sistemas son descritos por la mecánica hamiltoniana, originalmente desarrollada para describir la dinámica de los cuerpos celestes. La terminología de la teoría del caos se refiere a estos sistemas como sistemas hamiltonianos. Los sistemas hamiltonianos tienen un subsistema conocido como sistema ergódico: "In 1990, Hilger initiated the theory of time scales as a theory capable of treating continuous and discrete analysis in a consistent way, based on which some authors have studied some Lyapunov inequalities for dynamic equations on time scales during the last few years" (Sun, Zeng, Su, Qin, 2018: 848). En los sistemas no disipativos el volumen no crece mientras que en los sistemas disipativos el volumen aumenta; y esta es la diferencia fundamental entre los dos sistemas.

Si quisiéramos definir la teoría del caos determinista dentro de la clasificación de las ciencias, deberíamos decir que es la llamada teoría ergódica de los sistemas dinámicos. "In recent years, singular perturbation problems without 
periodicity have also been a subject of interest in the context of mathematical finance. In those models, the analysis of ergodic problem (EP) is one of the main issues" (Ichihara, 2015: 626). El Sistema dinámico es un sistema en el cual el comportamiento puede ser modelado por medio de un sistema de ecuaciones diferenciales: "It is well known that chaotic dynamics is not restricted only to complicated and strongly nonlinear vector fields but can be observed also in the case of algebraically simple systems with six terms including nonlinearity" (Gotthans, Petrzela, 2015: 1143). Los sistemas dinámicos incluyen también la mecánica newtoniana clásica descrita por los hamiltonianos. El caos determinista puede ser considerada una contradictio in adiecto; sin embargo, permite también cierta libertad dentro de cierto intervalo de tiempo.

\section{La física del caos}

Ahora bien, no hay que olvidar que el caos perfecto es una abstracción. Hay situaciones en las cuales es realmente imposible determinar las consecuencias de una causa. Es posible hacerlo de manera retrospectiva, pero a menudo es imposible determinar las consecuencias de antemano en una línea de tiempo, ya que la previsibilidad es limitada. Si ocurre el caso mencionado, hablamos de una catástrofe; es decir, un cambio a peor repentino de las cosas, en el sentido etimológico de la palabra. Es cierto que el comportamiento de los sistemas caóticos es difícil de predecir en general; sobre todo en perspectivas a más largo plazo. Sin embargo, como demuestra la práctica, es posible influir en él hasta un cierto punto; entonces se puede moderar, cambiar, regular o estabilizar (Datcu, Stanciu, 2016).

A nivel micro, el caos está presente en la física cuántica. La física cuántica, en función de su interpretación, distingue dos opiniones sobre el caos. Muchas veces aparece la llamada Interpretación de Copenhague, que representa una de las interpretaciones de su funcionamiento. Naturalmente, las hipótesis clásicas derivadas de la física tradicional están en vigor con un alcance limitado. Todos los atributos derivados de una relación parcial onda-partícula no pueden ser aplicados a la física cuántica, ya que algunos de los atributos son incompatibles entre sí. El principio de indeterminación (o de incertidumbre) de Heisenberg habla sobre ellos (Roura , 2017). Esta teoría limita los conceptos de la física clásica. En la física clásica, la previsibilidad (o predictibilidad) funciona bastante bien. Sin embargo, es la relación de indeterminación la que indica sus considerables limitaciones.

Con respecto a cualesquiera sistemas caóticos deterministas, se dan consecuencias interesantes derivadas directamente de la imprevisibilidad. La imprevisibilidad es causada por la existencia de fluctuaciones. Las fluctuaciones pueden conducir a un desarrollo inescrutable del sistema. Los sistemas disipativos y no disipativos concuerdan en que puede ocurrir una situación cuyo desarrollo sería imposible de predecir. Básicamente, el nivel microscópico nos dice lo mismo. E1 caos en la mecánica cuántica sobresale por su imprevisibilidad, lo cual es percibido en la interpretación de Copenhague como una característica inmanente del sistema. Aunque las condiciones sean muy bien conocidas, no se puede predecir lo que ocurrirá en el futuro. La interpretación de Copenhague subraya inequívocamente este hecho.

También hay otras interpretaciones que participan de la física clásica sin rechazar el aparato matemático actual. La más conocida de estas interpretaciones es la de Louis de Broglie (en su versión original) y la de Bohm, que todavía destaca por su influencia. La introducción de los parámetros ocultos de Bohm, supone la posibilidad de una especificación precisa del comportamiento del sistema observado, bajo la condición de que el status quo inicial del sistema sea conocido. Esta interpretación,

XLinguae, Volume 10, Issue 4, October 2017, ISSN 1337-8384, eISSN 2453-711X 
junto con las interpretaciones de Bopp, Janossy y otros, supone la previsibilidad exacta de cálculo del desarrollo futuro de los objetos observados. Sin embargo, hay que añadir que estas interpretaciones sólo suponen y buscan los parámetros ocultos y que no son apoyadas por la mayoría de los físicos. La interpretación de Hugh Everett tampoco niega la previsibilidad. El presente estudio no analiza su interpretación con más detalle, aunque es muy interesante. Los físicos criticaron esta teoría por su postulación de múltiples mundos posibles y, seguramente, Guillermo de Ockham nunca apoyaría esta interpretación. Penrose en su interpretación defiende la creencia determinista; sin embargo, su teoría está abierta, hasta el punto de que permite también la versión indeterminista. Los marxistas defienden la previsibilidad, pero principalmente por razones más ideológicas que físicas. Hay muchas opiniones acerca de la previsibilidad de comportamiento de los objetos en el microcosmos que se contradicen a menudo entre sí. No obstante, en el caso de la física cuántica se puede afirmar que "the conflict of quantum physics interpretations continues" (Rau, 2017).

En la valoración del caos determinista, ya se ha dicho que no está claro si se trata del caos fundamental o caos causado por la sensibilidad extrema a las condiciones iniciales; es decir, si el caos no fundamental, sino práctico, sería prácticamente impredecible.

Desde una perspectiva estrictamente metódica, hay cuatro opciones: el caos cuántico y el caos determinista, en principio imprevisibles; el caos determinista y el caos de la mecánica cuántica, en principio previsibles; y sólo una especie de caos en principio previsible. Independientemente de si la previsibilidad es principal o no, se trata de un sistema extremadamente sensible.

\section{2. ¿Determinismo o indeterminismo?}

La teoría del caos está inseparablemente conectada con las cuestiones de la libertad de elección. El caos y las cuestiones de la libertad de elección se conectan principalmente a través de determinismo e indeterminismo. En el contexto del presente trabajo, definimos el concepto de determinismo como previsibilidad y el indeterminismo como imprevisibilidad. La física contemporánea ofrece sólo una respuesta ambigua a estas preguntas. No es posible llegar a una respuesta definitiva y exacta. Las respuestas están marcadas por la dicotomía, tanto a nivel micro como a nivel macro.

Desde el punto de vista matemático, hay una probabilidad de $75 \%$ de la existencia del indeterminismo; pero este hecho no tiene mucho valor informativo en sí. Lo más importante es que el aspecto de la libertad de elección se puede ver también a través de la cuestión de la sensibilidad del sistema. Es evidente que el caos determinista se caracteriza por su alta sensibilidad. "The inaccuracy of determining the initial data leads to a loss of relationship with the initial conditions" (Kachanov , 2013: 40) No es posible llegar a una predicción a más largo plazo, puesto que no tendría ningún sentido. Como hay innumerables imperfecciones en los sistemas reales, y cada uno de estos es capaz de cambiar el estado macroscópico del sistema, lógicamente ya no es posible predecir estos estados. (Medvinsky, Nurieva, Rusakov, Adamovich, 2017). El sistema es muy sensible y el conocido efecto mariposa apoya esta afirmación.

En este punto, es necesario introducir un postulado metafísico: el libre albedrío del ser humano. El presente estudio no va a demostrar el libre albedrío del ser humano; se considera un postulado metafísico de manera semejante al de la ética de Kant. Si queremos expresarnos de una forma cartesiana tradicional, el libre albedrío del ser humano significa que uno es libre de transferir esta libertad en el mundo físico, en el sentido del movimiento libre del cuerpo humano. En tal caso, podemos producir cambios considerables. No vivimos en un mundo sobre el que no 
podemos influir. La gente no vive en un mundo en el que es difícil influir; no obstante, su comportamiento puede tener consecuencias importantes, ya que puede afectar notablemente al mundo. Por otro lado, de la naturaleza del caos resulta que el mundo es muy inestable y no se puede prever nada a largo plazo. Esto parece ser un argumento en contra de los metarrelatos predichos a largo plazo por la historia.

Hay que añadir que lo anteriormente expresado causa preocupación en las ciencias sociales. Además, los fenómenos naturales determinan lo social; o al menos lo condicionan. Esto implica una doble responsabilidad. Existe una responsabilidad derivada del argumento de que estos sistemas son muy inestables; es decir, la gente influye en gran medida sobre los acontecimientos en el mundo. Por otro lado, debido a la inestabilidad de estos sistemas, la responsabilidad resulta también del hecho de que los seres humanos no pueden confiar en la estabilidad en general, ni en la estabilidad de las acciones ya realizadas. El ser humano con libre albedrío es responsable de provocar acontecimientos en el mundo que permiten los cambios físicos. Tomar decisiones implica también decidir sobre los cambios que hacemos. Como estos cambios no son estables, es necesario tomar decisiones con bastante frecuencia, lo que va de la mano con la responsabilidad por los cambios realizados. Cambiar significa decidir, y decidir significa aceptar una gran responsabilidad. Es necesario enseñar a la gente a ser responsable, ya que en la actualidad se pone el acento cada vez más en la importancia de la educación y autoeducación que desembocan en un desarrollo sano de la persona (Tao, Yu, Gao, Xue, 2016).

Desde el punto de vista de la cosmología moderna, la libertad tiene también consecuencias éticas muy importantes. Si supusiéramos que el concepto de creatio ex nihilo es confirmado a través de la cosmología, no sería posible decirlo así, a pesar de ciertos indicios. "It is well known that wave propagation in random media can be described in the framework of the Fokker-Planck equation, under certain conditions" (Iomin, 2017). El problema se basa en la existencia del Tiempo de Planck, conocido también como el Muro de Planck, el cual impide la descripción de cualquier cosa. Hay muchas discusiones respecto a lo que precedía al Tiempo de Planck, pero ¿es realmente científico explotarlo? No es posible hablar de los eventos acontecidos antes del Tiempo de Planck. En efecto, la cosmología nos puede llevar casi al origen del universo, al tiempo cercano a lo que se supone ser el origen del universo. Sin embargo, se trata sólo de e speculaciones y no de serias declaraciones científicas. En la actualidad, un modelo teórico reconocido generalmente como estándar, desde el punto de vista de la cosmología, no puede analizar de una manera infalible lo que realmente ocurrió antes del Tiempo de Planck. Los científicos consideran la aplicación de teorías infalibles en el tiempo precedente al comienzo de la inflación $\left(10^{-35} \mathrm{~s}\right)$ como problemática. En este punto todas las informaciones operadas por la física son imprecisas.

La física puede describirse a sí misma sólo de acuerdo con su propio paradigma y en conformidad con las cuestiones científicas. La física no puede ser la metateoría de la ciencia. Debido a ciertas limitaciones, la física no puede describir ciertos hechos, y seguramente no puede describir la situación precedente al Muro de Planck. La cosmología no es capaz de describir las condiciones precedentes a la Gran Explosión (Big Bang). La cosmología física puede describir muchas cosas; ahora bien, no puede explicar la creación del universo. Operamos con las conclusiones y posiciones abiertas. La posibilidad sigue siendo dicotómica: el mundo existía antes de la inflación y no podemos hablar del origen en el tiempo, o el origen simplemente no existe. Kant en su idea cosmológica reconoce que existe la aporía sin respuesta. El mundo no puede ser considerado como finito o infinito; ni siquiera es posible si ponemos la flecha del tiempo al revés. 


\section{Implicaciones éticas y religiosas}

Es imposible decidir cuál de las opciones debería ser elegida. La cosmología física dice básicamente lo mismo. El paradigma actual no lo permite, porque se rebasaría el alcance de las competencias de la física como una ciencia natural fundamental. Las consecuencias éticas del problema radican en la eliminación de cualquier opinión fundamentalista. El mismo principio antrópico habla a favor de la intervención inteligible; no obstante, eso no se puede considerar como evidencia, a pesar de que los indicios son muy fuertes. Y aunque existen indicios significativos, no pueden evidenciar la influencia de un ser superior sobre la creación del hombre. Por otra parte, estos indicios son muy importantes para aquellos que creen en dicha solución. De acuerdo con la actitud de fe de Wittgenstein, es un indicio para aquellos que deciden creer en algún tipo de intervención de un poder superior. Sin embargo, su decisión se basa en su libre albedrío, no en las evidencias concretas.

En lo que se refiere a las cuestiones religiosas, no es posible predecir nada concreto. La cosmología física todavía no ha respondido a la pregunta fundamental, de modo que no ha facilitado ninguna respuesta a la religión o a la filosofía. La curiosa pregunta de la teología natural (theologia naturalis) todavía no tiene ninguna respuesta satisfactoria. No son palabras huecas. De lo anterior resulta que no somos capaces de elaborar un sistema holístico racional que podría hacer encajar las preguntas éticas con las preguntas metafísicas, en el sentido de que la ciencia misma apoyaría este sistema de una manera consistente e irreversible. Por lo tanto, la consecuencia ética es la abstención intelectual, en el sentido de abstenerse de emitir juicios que resulten directamente de la representación cosmológica del mundo. Desde el punto de vista de la cosmogonía, no es posible formular ninguna conclusión clara que hable a favor de la creación del mundo o a favor de la imposibilidad de su creación. El impacto religioso de la descripción cosmológica de la creación del mundo es neutral. Lo cual representa también una base para una orientación ética claramente neutral que no favorezca a ningún sistema filosófico o religioso que exprese opiniones concretas en esta materia. Consecuentemente, el sistema de normas éticas no se puede basar en los principios que provienen de los resultados de la cosmología. Pero si eso es así, ¿qué tipo de fundamentación puede tener? Pensamos que solo sería posible en una antropología en la que el ser humano se constituyera como dotado de valor en sí mismo, y que tuviera en cuenta tanto su determinación biológica, como la sociocultural y espiritual.

¿Qué resulta de lo anterior? Con respecto a la ética, la consecuencia es el derecho de todos a tener su propia creencia religiosa y filosófica. Por otra parte, las conclusiones anteriores rompen con cualquier argumento de las posiciones fundamentalistas. Estas conclusiones se pueden ver como un camino para las posiciones tolerantes, no fundamentalistas. Así pues, en este contexto, con el paradigma actual y con el nivel actual de conocimientos, apoyamos la opinión de que es un resultado positivo ético de la cosmología física el hecho de que no sea posible imponer ningún sistema filosófico, religioso o ideológico. Sin embargo, hay que dejar claro que la neutralidad ética solo es posible si hacemos abstracción de las implicaciones éticas de la labor del científico que, como ser humano, le son ineludibles. Por su parte, las creencias religiosas pueden jugar tanto un papel positivo como negativo, en la medida en que engrandezcan o no la dignidad humana y el respeto a los derechos humanos.

Desde el punto de vista de la física, la altamente probable libertad de acción, metafísicamente supuesta por Kant como el libre albedrío del ser humano, tiene adicionalmente sus consecuencias éticas. Como la libertad existe en el nivel de la física, no se debería interferir en la expresión de las actitudes fundamentales ideológicas; especialmente cuando la cosmología física no ofrece ninguna evidencia convincente, a pesar de algunos indicios. 
Dicho con otras palabras: "It is necessary to point out the difference between the general concept of ethics and the Christian concept of ethics". (Rusu, 2013). Por consiguiente, es necesario diferenciar entre cómo uno debería actuar como cristiano y cómo debería actuar como un hombre con una opinión filosóficamente independiente, de acuerdo con la unidad interpersonal de una acción. En este caso, los principios éticos indican el carácter natural de la libre elección ideológica, teniendo en cuenta el escepticismo ideológico metódico, así como los resultados de la filosofía y las ciencias presentes hasta hoy. Los principios de la teología moral subrayan que la fe es una donación de Dios. La fe puede tener diversas intensidades y debe ser difundida a través de la evangelización de forma altruista; sin embargo, nunca por la fuerza. La reflexión íntima sobre todo el sistema del mundo debe originarse como el resultado de la libertad, no de la violencia.

Incluso si aceptamos la interpretación determinista al nivel del macro y microcosmos, el caos determinista tiene rasgos de una gran sensibilidad sistémica. Por ello, incluso un pequeño impulso puede provocar que el sistema sea impredecible. En tal caso, hay muchas opciones en cuanto a los cambios; y de ello resulta también la existencia de muchas opciones relacionadas con las intervenciones de Dios en este mundo. Imaginemos, por ejemplo, la intervención por medio de los organismos vivos. Si nosotros, al igual que Kant, reconocemos que el libre albedrío del hombre es un postulado metafísico, y si también reconocemos la interpretación de Agustín de Hipona de la gracia de Dios, dentro del marco de la filosofía cristiana, entonces podemos considerar la influencia de Dios sobre el hombre como un espacio posible para la influencia de Dios en el mundo; siempre que seamos conscientes de las consecuencias prácticas del caos determinista.

\section{La fe en la razón y la razón de la fe}

Finalmente, quizás sea oportuno replantearse una cuestión que, aunque antigua, sigue teniendo importancia: ¿cómo compaginar razón y fe? Evidentemente puede haber, y hubo, varias maneras de responder a tales cuestiones. La más coherente e ideal fue la de Agustín de Hipona, que identificó filosofía y religión (García-Junceda, 1986, 103-118). La postura extrema a esta fue la de Guillermo de Okham, que las separó totalmente; mientras que la postura intermedia de Tomás de Aquino estuvo en distinguirlas, aunque con ámbitos comunes. En S. Agustín el problema de las relaciones fe-razón fue el problema de su existencia, el problema de Agustín mismo. En cambio, en Sto. Tomás fue el problema de las relaciones entre las ciencias Filosofía y Teología. Por último, en Okham se trataba - a nuestro parecer- del problema de las relaciones Estado-Iglesia. Otra postura más moderna fue la de Espinosa, que defendió también la separación, aunque no la oposición, de fe-razón o religión-filosofía. Para Espinosa tal separación se basa en los distintos fundamentos y fines de ambos, y en último término se debe a su preocupación político-religiosa por la salud del Estado (Spinoza, 1986). En general, la filosofía moderna no es sino la consecuencia lógica de la crisis de la metafísica (como estudio del ser y de Dios) iniciada ya con la doctrina de la doble verdad averroísta, y que se manifestará de manera plena con la negación del orden trascendental y la concepción de la esencia divina como infinitud en el idealismo alemán (Falgueras, 1992: 133-152).

En cuanto a Kierkegaard, coincide con la tradición católica en afirmar que la verdad fundamental para el hombre es aquella que ofrece una salvación eterna, que ésta implica la fe en los misterios divinos, y que conlleva, pues, traspasar los límites de la razón. (Milic, Repar, 2017; Stur, Mitterpach, 2017). Sin embargo, divergen respecto a la conclusión: para Kierkegaard sobrepasar esos límites lleva a la paradoja, al absurdo y al escándalo, lo cual no lo comparte el catolicismo (Guerrero, 1991: 990992). Para el escritor danés, en fin, la razón no puede ayudar "positivamente" a la fe;

XLinguae, Volume 10, Issue 4, October 2017, ISSN 1337-8384, eISSN 2453-711X 
sólo tendría una función "negativa", esto es, señalar los límites de la misma razón respecto a la fe. En este sentido, Kierkegaard fue un postmoderno (Guerrero, 1991: 990-992) en pleno siglo XIX. Más allá de estos planteamientos teóricos, para el danés es esencial acomodar la comunicación de la verdad existencial (o subjetiva) a la comunicación de poder; de lo contrario, la fe se transmitiría erróneamente como una comunicación de saber (Kierkegaard, 2017).

Ahora bien, no debemos pensar que este tema es una cuestión meramente académica. Al contrario, se halla en nuestra vida diaria, en nuestro mundo más cercano. ¿Cuántas veces, si no, damos fe de algo? ; o ¿cuántas veces damos crédito a lo que nos han dicho, vemos o conocemos?; ¿no son, en último término, los conocimientos científicos una cuestión sobre la que debemos prestar nuestro asentimiento (Bruckner, 1998: 64), aunque puedan ser ínter subjetivos?; aún más, ¿depende la fe (sea la que sea) de los hechos mismos, o no es más bien una cuestión personal y subjetiva que para nada influye la constatación puramente objetiva de los mismos, sino que quizá debemos pasar por encima de ellos, ir más allá o apostar por lo absurdo e irracional - tal como afirmaría también Kierkegaard? Pensamos que queramos o no, seamos religiosos o no, la fe juega un papel muy importante en nuestras vidas. Tanto que me atrevería a afirmar que sin fe la vida misma no vale nada; porque, ¿cómo existir sin fe en uno mismo y en lo que hace? Creemos en las cosas, en la gente, más de lo que pensamos. Sin embargo, no siempre somos conscientes de ello.

\section{Conclusión}

Normalmente y de forma errónea identificamos la verdad con la demostración científica, cuando - si somos rigurosos - la razón ${ }^{1}$ científica es bastante estrecha e insatisfactoria. Así, se pretende fundamentar de forma objetiva esa certeza y seguridad en nuestros conocimientos; pero dichos estados son subjetivos y como tales depende por entero del individuo, y del significado que tengan en su existencia. Al final, lo que parece realmente importante es mi convicción propia respecto a lo que sea la verdad, porque será esto lo que determine mi existencia como individuo. Por tanto, la fe es una opción y una necesidad personal que poco tiene que ver con lo estrictamente racional, lo cual no quiere decir que no sea una cosa razonable. Queremos decir que debe diferenciarse entre lo racional como aquello que se sustenta en la razón humana y sus valores, y lo arracional, que es aquello que está completamente fuera de lo racional. Podemos entender la fe como algo irracional, pero no como algo que no sea razonable, o que no tenga nada que ver con la razón humana y sus valores. En este sentido, conviene abrirse a otras dimensiones no racionales en el hombre, aunque no por ello menos humanas. No obstante, para ello se requiere una mirada más profunda sobre la realidad, una visión más lejana y dar ese salto (como proponía Kierkegaard) hacia la fe. Lo malo de todo esto es ese vértigo que produce ver el vacío ante nuestros pies, y no tener en su totalidad la seguridad de que lleguemos a alguna parte: tener fe es poder caminar sobre las aguas de nuestro destino y no hundirnos. La razón no es sino esa barca en la que pretendemos sentirnos seguros, desconociendo el viento que sopla en las velas y la inmensidad del océano. Y la libertad sería el timón como la condición de posibilidad de que nuestra voluntad pueda dirigirnos hacia la tierra de la verdad y la felicidad, más allá de lo contingente y efímero.

Esta contribución es fruto del proyecto Consecuencias Éticas y Sociales de Problemas 
Seleccionados de las Ciencias Naturales IG-KSV-01/2016 / 2.1.5 and Slovak Research and Development Agency under the contract No. APVV-16-0016".

\section{Bibliographic references}

BALLESTEROS, J. 1989. Postmodernidad: decadencia o resistencia. Madrid, Tecnos (18).

BRUCKNER, P. 1998. La tentación de la inocencia, Barcelona, Círculo de Lectores (20).

DATCU, O. - STANCIU, M. 2016. Chaotic synchronization signal shape alteration as seen by a higher-order sliding-mode observer, In: Revista Tecnica de la Facultad de Ingenieria Universidad del Zulia 39 (1).

FALGUERAS SALINAS, J. I. 1992. "Los orígenes medievales de la crisis de la metafísica", In: Thémata, Revista de Filosofía, nº 9, (16).

GARCIA-JUNCEDA, J. A. 1986. La cultura cristiana y S. Agustín. Madrid, Cincel (14).

GARCIA MARTIN, J. - GIORDANO, D. 2011. Kierkegaard y la comunicación. Napoli, Orthotes Editrice (19).

GISSINGER, C., 2012. A new deterministic model for chaotic reversal, In: The European Physical Journal B 85 (4).

GOTTHANS, T., - PETRZELA, J. 2015. New class of chaotic systems with circular equilibrium, In: Nonlinear Dynamics 81 (3).

GUERRERO MARTINEZ, L. I. 1991: "Fe luterana y fe católica en el pensamiento de Kierkegaard", in:, Scripta Theologica 3, Pamplona (17).

HAJKO, D. 2011. Vrucnost a cin alebo Kierkegaardovske meditacie. Bratislava: Hajko \& Hajkova. 136 p. ISBN 978-80-88700-78-4.

Ichihara, N., 2015. The generalized principal eigenvalue for Hamilton-Jacobi-Bellman equations of ergodic type, In: Annales de l'Institut Henri Poincare (C) Analyse Non Lineaire 32 (3).

Iomin, A., 2017. Subdiffusion in classical and quantum nonlinear Schrödinger equations with disorder, In: Computers and Mathematics with Applications 73 (6).

KACHANOV, Y. S., 2013. Hypothesis on deterministic turbulence, In: European Journal of Mechanics, B/Fluids 40.

KHAN, A. 2017. Ochrana pred nepochopenim pri filozofovani nad Kierkegaardom. Vo vystavbe. In: Kierkegaard and the Crisis of the Contemporary World. Acta Kierkegaardiana- Supplement. Vol. 6, Ed. Roman Kralik et al. Toronto - Nitra: Kierkegaard Circle, University of Toronto. pp. 26-42. ISBN: 978-0-9878168-4-9.

KIERKEGAARD, S. 2017. La dialéctica de la comunicación ética y ético religiosa (Den Ethiske og den Ethiske-Religieuse Meddelelses Dialektik [1847]). In: Argumenta philosophica, Barcelona (19).

KONDRLA, P. - REPAR, P. 2017a. Ontological consequences of the ethics of technology. In: Komunikacie, vol. 19, n. 1, pp. 19-24. ISSN 0341-2059.

KONDRLA, P. - REPAR, P. 2017b. Postmodern aspects of new religious movements. In: European Journal of Science and Theology, vol. 13, n. 3, pp. 67-74. ISSN 1841-0464.

KONDRLA, P. - TOROK, L. 2017. Objective faith and weak truth. In: European Journal of Science and Theology, vol. 13, n. 1, pp. 79-86. ISSN 1841-0464.

LLANO, A., 1988. La nueva sensibilidad. Madrid, Espasa (18).

MAHRIK, T. 2015. The death of Philosophy in Kierkegaard and Hawking. In: European Journal of Science and Theology, vol. 11, n. 5, pp. 23-32. ISSN 1841-0464. MAHRIK, T. Kierkegaardian pointers to metaethics. 2017 Source of the Document In: European Journal of Science and Theology, vol. 13, no. 5, pp. 119-130. ISSN 1841-0464.

XLinguae, Volume 10, Issue 4, October 2017, ISSN 1337-8384, eISSN 2453-711X 
MARTIN, J. G. 2017. The category of the single individual in Kierkegaard. In: European Journal of Science and Theology, vol. 13, n. 3, pp. 99-108. ISSN 18410464.

MEDVINSKY, A.B. - NURIEVA, N.I. - RUSAKOV, A.V. - ADAMOVICH, B.V. 2017. Deterministic chaos and the problem of predictability in population dynamics, In: Biophysics (Russian Federation) 62 (1).

MILIC, U. - REPAR, P. 2017. Singularity and the existential turn. In: European Journal of Science and Theology, vol. 13, n. 3, pp. 109-119. ISSN 1841-0464.

PAVLIKOVA, M. 2017a. Consciousness of Anxiety in Literary Work of Don DeLillo. In: XLinguae, vol. 10, n. 1, pp. 62-69. ISSN 1337-8384.

PAVLIKOVA, M. 2017b. Kierkegaard's Reflection in Don DeLillo's Novel 'Falling Man'. In: European Journal of Science and Theology, vol. 13, n. 1, pp. 15-23. ISSN 1841-0464.

PAVLIKOVA, M. 2017c. Reading Auden as a resource for existential reflection in a society with technocratic and hedonistic tendencies. In: Komunikacie, vol. 19, n. 1, pp. 39-43. ISSN 0341-2059.

PAVLIKOVA, M. 2016. The concept of anxiety and its reflection in Auden's work 'the Age of Anxiety' In: European Journal of Science and Theology, vol. 12, n. 4, pp. 111-119. ISSN 1841-0464.

RAU, A. R. P. 2017. What is physics? The individual and the universal, and seeing past the noise, In: Physics Essays 30 (1).

ROURA, A. 2017. Circumventing Heisenberg's Uncertainty Principle in Atom Interferometry Tests of the Equivalence Principle, In: Physical Review Letters 118 (16).

RUSU, I. 2013. Religious freedom, In: European Journal of Science and Theology 9 (6).

SLIVKA, D. - MIERZWA, J. 2017. Intentional abuse of social networks with the goal of promoting ideas of anti-semitism, racism, and xenophobia. In: Komunikacie, vol. 19, n. 1, pp. 54-58. ISSN 0341-2059.

SLIVKA, D. Hebraic scriptural hermeneutics in the ancient Hellenistic world as the basic approach of early Christians. In: European Journal of Science and Theology, vol. 11, no. 5, pp. 87-96. ISSN 1841-0464.

SPINOZA, B., 1986. Tratado teológico-político. Traducción, introducción, notas e índices de Atilano Domínguez. Madrid, Alianza Editorial, (15).

STUR, M. 2011. Responsibility and sensus communis at Unamono's, Ortega y Gasset's and Heidegger's work. XLinguae, vol. 4, n. 4, pp. 30-39. ISSN 1337-8384.

STUR, M. - MITTERPACH. 2017. Different and identical features of the philosophical, scientific, artistic and religious knowledge in the context of Kierkegaard's thought. In: European Journal of Science and Theology, vol. 13, n. 1, pp. 35-46. ISSN 1841-0464.

SUN, T. - ZENG, F. - SU, G. - QIN, B. 2018. Lyapunov inequalities of quasihamiltonian systems on time scales, In: Journal of Computational Analysis and Applications 25 (5).

TAO, S. - YU, L. - GAO, W. - XUE, W. 2016. Food preferences, personality and parental rearing styles: analysis of factors influencing health of left-behind children, In: Quality of Life Research 25 (11).

TAVILLA, I. 2017. Some notes about the biblical influence on Kierkegaard's theory of stages. In: European Journal of Science and Theology, vol. 13, n. 1, pp. 87-94. ISSN 1841-0464.

TURCAN, C. 2017. Rousseau and Kierkegaard authenticity of human existence. In: European Journal of Science and Theology, vol. 13, n. 1, pp. 5-13. ISSN 1841-0464. VALCO, M. 2016. Koncepcia subjektu a viery u S. Kierkegaarda a D. Bonhoeffera. Eticke implikácie. Ljubljana: KUD Apokalipsa. 122 p. ISBN 978-9616-89-479-1. 
VALCO, M. 2017. The value of Dietrich Bonhoeffer's theological-ethical reading of Soren Kierkegaard. In: European Journal of Science and Theology, vol. 13, n. 1, pp. 47-58. ISSN 1841-0464.

VALCOVA, K. - PAVLIKOVA, M. - ROUBALOVA, M. 2016. Religious existentialism as a countermeasure to moralistic therapeutic deism. In: Komunikacie, vol. 18, n. 3, pp. 98-104. ISSN 0341-2059.

VINTAR, S. - SIMEUNOVIÆ, L. G. 2017. Definition frames as language-dependent models of knowledge transfer, In: Fachsprache 39 (1-2).

ZALEC, B. 2017. Preferential and non-preferential love in light of Kierkegaard's thought. In: European Journal of Science and Theology, vol. 13, n. 2, pp. 179-189. ISSN 1841-0464.

ZALEC, B. 2014. Nazism and Stalinism in the light of Kierkegaard's thought. In: Filozofia, vol. 69, n. 5, pp. 443-450. ISSN 0046-385X.

Words: 5536

Characters: 35481 (19,71 standard page)

Marián Ambrozy, PhD.

College of International Business ISM

Duchnovicovo námestie 1,

Prešov 08001

Slovakia

ambrozy.marian@gmail.com

Prof. Dr. Roman Králik, ThD.

Faculty of Arts, Constantine the Philosopher University in Nitra,

Hodzova 1, 94901

Nitra

Slovakia

rkralik@ukf.sk

Prof. Dr. José Garcia Martin

University of Granada, Department of Sociology,

Faculty of Political Sciences and Sociology, Calle Rector López Argüeta s/n, Granada,

Spain

joeg.martin10@gmail.com 